\title{
The Impacts of Harvesting Activities on Prey-Predator Fishery Model in the Presence of Toxin
}

\author{
Tau Keong Ang*, Hamizah M. Safuan and J. Kavikumar \\ Department of Mathematics and Statistics, Faculty of Applied Sciences and Technology \\ Universiti Tun Hussein Onn Malaysia, 86400 Parit Raja, Johor, Malaysia
}

Received 30 September 2017; accepted 23 May 2018; available online 1 August 2018

DOI: https://10.30880/jst.2018.10.02.021

\begin{abstract}
The present paper discusses a prey-predator fishery model where both species are subjected to harvesting effort. Both species release some toxic substances to each other. The prey species obeys the law of logistic growth. The model is a modified version from the classic Lotka-Volterra predator-prey model. The equilibria of the model are obtained and the dynamical behaviors of the proposed system are examined. Simulations of the model are performed and bifurcation diagrams are studied. The effects of harvesting and toxin on the stability of steady states are examined. In the present research, we found that the impact of harvesting activities is more influential on the dynamical behaviors in the fishery model than that of the toxin.
\end{abstract}

Keyword: Harvesting; Fishery; Toxicity; Predator; Prey.

\section{Introduction}

The on-going civilization and urbanization have expedited the process of exploitation of natural resources and altered the environment. In the recent years, there is a substantial growth of global concerns on the impacts of harvesting activities and toxic chemical substances on organisms and ecosystems. One of the significant apprehensions is the anthropogenic toxin released by the industrial waste to aquatic environments. According to the online database of American Fisheries Society, there are approximately 250,000 new chemical compounds manufactured every year for various demands. Due to the bioaccumulative and non-degradable properties of some chemical substances, they turn into toxin chemical substances and are released into aquatic environment. The consequent effects will affect the aquatic organisms including the fish species.

Mathematical modelling on population models has been developed to study the ecotoxicological problems. One of the leading studies is the toxicant-population model developed by Hallam et al. [1]. Hallam studied the effects of toxicants on the population by considering the environmental uptake rate of toxicants, loss rate of toxicants and body burden of the population. Hallam et al. [2] enhanced the model by employing the concept of first order kinetics on the concentration of toxicants in an organism. Both chronic and acute levels of toxicants were discussed in terms of persistence and extinction of the population. Similar research was done by $\mathrm{Pal}$ and Samanta [3] where they studied the model using bifurcation analysis and found periodic solution. Hallam and De Luna [4] investigated the impacts of toxicant on population where the population are exposed to both environmental and food chain platforms. Both studies by Hallam emphasized on the toxicantpopulation interaction of a single population.

Freedman and Shukla [5] proposed a toxicant-dependent model in single species by involving a prey-predator interaction. Besides, they also investigated the effects of toxicant on the species by considering the toxicant dependent carrying capacity. A two species competitive model was developed by Chattopadhyay [6] to examine the impacts of toxic substances released by each species to other species. Similar work was done by Samanta [7] to study the dynamical behaviors of a two competing species in the presence of toxicants.

Since most of the models incorporating toxicants only emphasize on general species, many research are now working on models in a more specific terrestrial such as the fishery 
model. Huang et al. [8] studied a toxindependent model on aquatic population to probe the direct effects of toxin (mercury) on the rainbow trout population. A research on the fish behaviors obstructed by the environmental contaminants was carried out by Scott and Sloman [9] to magnify the social behaviors of fish population such as the reproductive, mortality and metabolic rates due to the toxicant exposure.

On the other hand, many researchers work towards the influence of toxicants on the preypredator interactions. Huang et al. [10] formulated a prey-predator model by taking into accounts the influence of both environmental toxicants and toxicants from food consumption. It was found that different exposure rates of prey and predator species to toxicants will lead to different long-term consequences.

Besides that, researches on the effects of harvesting in fishery models are becoming more valuable. One of the research work focusing on the harvesting in prey-predator model is the study of Hoekstra and Bergh [11] that considered three possible cases: no harvesting, periodic harvesting and maximal harvesting and their influences in the perspectives of economic views. The efforts of Kar [12] are to inculcate the concept of selective harvesting in a prey-predator model where there are some restrictions on the harvested species such as the size, weight and age of the targeted harvesting species. Selective harvesting was proved to be more sensible and advisable in bioeconomic aspects if compared to random harvesting. Besides, Mukhopadhyay and Bhattacharyya [13] proposed a model with two predators competing for a single prey species subjected to logistic growth to examine the effects of harvesting on one predator species to the stochastic equilibrium of the model.

There is no doubt that the presence of toxicity will pose some effects on the harvesting activities on fish population. Ghosh et al. [14] developed a prey-predator fishery model to study the relationship between the pollutant concentrations and the equilibrium of fish population where the predator species are subjected to harvesting activities. The studies of Das et al. [15] and Haque and Sarwardi [16] considered the prey-predator fishery models by assimilating the different toxicant exposure rates on predator and prey. Both predator and prey species are subjected to different harvesting efforts and activities. Slight different from the work of Das et al. [15] and Haque and Sarwardi [16], Kar and Chaudhuri [17], discussed the dynamical behaviors of two competitive fishery system. These models focused on the persistence, extinction, stability and optimal harvesting policy in the presence of toxicity.

The main concern of this paper is to study the dynamical behaviors of a predator-prey fishery model in the existence of toxin. The prey species obeys the logistic growth rate and both species undergo different harvesting rates. The effects of harvesting and toxicant on both species are examined to make further speculations on the persistence and extinction properties. Through stability analysis and bifurcation results, we had proved that the effect of harvesting activities is more prominent than toxin in the fishery model.

\section{Model Formulation}

The classical Lotka-Volterra model describes dynamical interaction between the predator and the prey species. The LotkaVolterra model [18] has the linear form of equations

$$
\begin{aligned}
& \frac{d X}{d t}=r_{1} X-\gamma_{1} X Y, \\
& \frac{d Y}{d t}=-r_{2} Y+\gamma_{2} X Y,
\end{aligned}
$$

where $X(t)$ and $Y(t)$ represent the prey and predator population size at time $t$ respectively. From the model, the prey population grows at a rate of $r_{1}$ without the presence of predator population while the predator population dies out at a rate of $r_{2}$ in the absence of prey. The parameters $\gamma_{1}$ and $\gamma_{2}$ indicate the respective rates of change of prey and predator due to predation.

Our main intention is to study the effects of toxin on a harvested fishery model. We assume that both species are inflicted by a harvesting effort and each species produce toxin to other species. By applying the logistic law of growth on prey population, a system of differential equations similar to Kar and Chaudhuri [17] is modeled as 


$$
\begin{aligned}
\frac{d X}{d t}= & r_{1} X\left(1-\frac{X}{K}\right)-\gamma_{1} X Y-a E X \\
& -b X^{2} Y \\
\frac{d Y}{d t}= & -r_{2} Y+\gamma_{2} X Y-c E Y-d X Y^{2}
\end{aligned}
$$

The parameter $K$ is the environmental carrying capacity of the prey population, meaning the prey population grows logistically in the absence of predator species. Both species are inflicted by a combined harvesting effort of $E$, where the catchability coefficients of prey and predator species are denoted by $a$ and $c$ respectively. The parameters of $b$ and $d$ indicate the toxicity coefficients of prey and predator species respectively. All parameters are assumed to be positive values.

\section{Non-dimensional model}

Non-dimensionalization is carried out on the system (2) in order to reduce the number of parameters and to simplify the model for better interpretation. By introducing the scaled variables

$$
x=\frac{\gamma_{2} X}{r_{1}}, y=\frac{\gamma_{1} Y}{r_{1}}, \tau=r_{1} t
$$

the dimensional system (2) becomes

$$
\begin{aligned}
& \frac{d x}{d \tau}=x(1-\alpha x)-x y-\beta x-\sigma x^{2} y, \\
& \frac{d y}{d \tau}=-\delta y+x y-\varepsilon y-\rho x y^{2},
\end{aligned}
$$

where

$$
\begin{aligned}
& \alpha=\frac{r_{1}}{K \gamma_{2}}, \beta=\frac{a E}{r_{1}}, \sigma=\frac{b r_{1}}{\gamma_{1} \gamma_{2}}, \\
& \delta=\frac{r_{2}}{r_{1}}, \varepsilon=\frac{c E}{r_{1}}, \rho=\frac{d r_{1}}{\gamma_{1} \gamma_{2}} .
\end{aligned}
$$

In system (3),

$\alpha$ : ratio of the growth rate of $x$ to the product of growth rate of $y$ by $x$ and environmental carrying capacity of $x$; $\beta$ :ratio of the product of catchability coefficient of $x$ and harvesting effort to the growth rate of $x$;

$\sigma$ : ratio of the product of coefficient of toxicity and the growth rate of $x$ to the product of growth rate of $x$ by $y$ and growth rate of $y$ by $x$

$\delta$ : ratio of the growth rate of $y$ to $x$;

$\varepsilon$ :ratio of the product of catchability coefficient of $y$ and harvesting effort to the growth rate of $x$;

$\rho$ : ratio of the product of coefficient of toxicity on $y$ and the growth rate of $x$ to the product of growth rate of $x$ by $y$ and growth rate of $y$ by $x$.

\section{Steady States, Equilibria and Stability Analysis}

In this section, we inspect the equilibria and the stability of system (3). One of the useful methods is by using the Jacobian matrix, where the system is generalized to

$$
\begin{aligned}
& J= \\
& {\left[\begin{array}{cc}
-2 x(\sigma y+\alpha)-\beta-y+1 & -x(\sigma x+1) \\
y(1-\rho y) & x(-2 \rho y+1)-\delta-\varepsilon
\end{array}\right]}
\end{aligned}
$$

The equilibria of system (3) are obtained by solving $\frac{d x}{d \tau}=\frac{d y}{d \tau}=0$. There are three possible steady states in the form of $P_{i}(x, y)$ where two of them are trivial steady states and one is nontrivial steady state, $i=1,2,3$. The two trivial states are

$$
P_{1}=(0,0) \text { and } P_{2}=\left(\frac{1-\beta}{\alpha}, 0\right) \text {. }
$$

The equilibrium $P_{1}$ represents the extinction of both predator and prey species. The Jacobian matrix at $P_{1}$ is $J_{\left(P_{1}\right)}=\left[\begin{array}{cc}1-\beta & 0 \\ 0 & -\delta-\varepsilon\end{array}\right]$, which gives the characteristic equation of

$$
\begin{aligned}
& \lambda^{2}+(\beta+\delta+\varepsilon-1) \lambda \\
& \quad+(\beta-1)(\delta+\varepsilon)=0
\end{aligned}
$$

where $\lambda$ is the eigenvalue. Equilibrium $P_{1}$ has a set of eigenvalues $E_{P_{1}}=\{-\delta-\varepsilon, 1-\beta\}$. We know that $-\delta-\varepsilon$ is always negative, thus $P_{1}$ is a stable node if $\beta>1$ or $P_{1}$ is an unstable saddle point if $\beta<1$.

On the other hand, equilibrium $P_{2}$ indicates persistence of the prey population in the 
absence of predator population. The Jacobian matrix at $P_{2}$ is

$$
J_{\left(P_{2}\right)}=\left(\begin{array}{cc}
\beta-1 & -\frac{\sigma(\beta-1)^{2}}{\alpha^{2}}+\frac{\beta-1}{\alpha} \\
0 & -\delta-\varepsilon-\frac{\beta-1}{\alpha}
\end{array}\right),
$$

that gives the characteristic equation of

$$
\begin{gathered}
\lambda^{2}+\left(\frac{\alpha+\alpha \delta+\alpha \varepsilon-\alpha \beta+\beta-1}{\alpha}\right) \lambda \\
+\frac{(1-\beta)(\alpha \delta+\alpha \varepsilon+\beta-1)}{\alpha}=0 .
\end{gathered}
$$

The set of eigenvalues is $E_{P_{2}}=\{\beta-1$, $\left.\frac{1-\alpha \delta-\alpha \varepsilon-\beta}{\alpha}\right\}$. Here, we consider the appropriate conditions:

$$
\begin{aligned}
\alpha \delta+\alpha \varepsilon+\beta & <1, \\
\alpha \delta+\alpha \varepsilon+\beta & >1, \\
\beta & <1, \\
\beta & >1 .
\end{aligned}
$$

If condition [i] holds, condition [iii] is guaranteed and thus both $P_{1}$ and $P_{2}$ are unstable saddle points. Thus, it is guaranteed that the non-trivial steady state is stable. If the conditions [ii] and [iii] hold, then $P_{1}$ is an unstable saddle point but $P_{2}$ is a stable node. However, if conditions [ii] and [iv] hold, then $P_{1}$ is a stable node and $P_{2}$ is a unstable saddle point.

The non-trivial steady state of system (3) which represents the coexistence of both prey and predator species is

$$
P_{3}=\left(\hat{x}, \frac{\hat{x}-\delta-\varepsilon}{\rho \hat{x}}\right),
$$

where $\hat{x}$ is a root by solving the quadratic equation of

$$
a_{1} \hat{x}^{2}+a_{2} \hat{x}+a_{3}=0
$$

with

$$
\begin{aligned}
& a_{1}=\alpha \rho+\sigma \\
& a_{2}=\beta \rho-\sigma \delta-\sigma \varepsilon-\rho+1, \\
& a_{3}=-(\delta+\varepsilon) .
\end{aligned}
$$

\section{Existence of Limit Cycle}

In order to verify whether system (3) possesses limit cycle or periodic solution, we apply the Bendixson-Dulac criterion. Consider the system (3)

$$
\begin{aligned}
& \frac{d x}{d \tau}=x(1-\alpha x)-x y-\beta x-\sigma x^{2} y \\
& =f(x, y), \\
& \begin{aligned}
\frac{d y}{d \tau} & =-\delta y+x y-\varepsilon y-\rho x y^{2} \\
& =g(x, y),
\end{aligned}
\end{aligned}
$$

and by applying the function of $\phi(x, y)=\frac{1}{x y}$, we have

$$
\begin{aligned}
& \frac{\partial(\phi f)}{\partial x}+\frac{\partial(\phi g)}{\partial y} \\
= & \frac{\partial}{\partial x}\left(\frac{1-\alpha x-\beta}{y}-\sigma x-1\right) \\
& +\frac{\partial}{\partial y}\left(-\frac{\delta+\varepsilon}{x}-\rho y+1\right) \\
= & -\frac{\alpha}{y}-\sigma-\rho<0 .
\end{aligned}
$$

Since $-\left(\frac{\alpha}{y}+\sigma+\rho\right)$ is always negative for all the positive parameters, there is no closed orbit or no periodic solution presents in model (3).

\section{Bifurcation Results and Analysis}

For the purpose of investigating the dynamical behaviors of the model (3), parameter variation technique is used with the assistance of numerical software XPPAUT. The steady state diagrams are obtained using the MATLAB software. For simplicity, we set the parameters $\alpha=0.1, \sigma=2, \delta=0.1$ and $\rho=2$. In this section, we examine the effects of the harvesting parameter on prey, $\beta$, in equation (3a). Fig. 1(a) and 1(b) display the steady state diagrams with respect to parameter $\beta$. For illustrating purposes, the solid lines depict the stable steady states while the dotted lines depict the unstable steady states. 


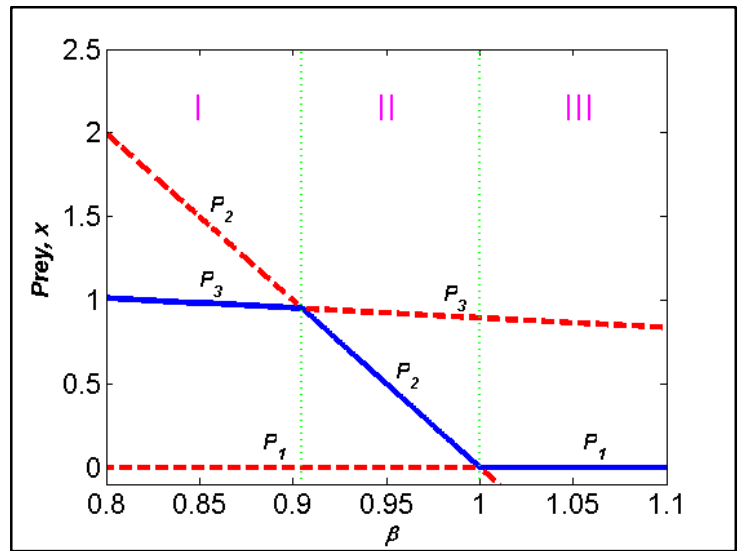

(a)

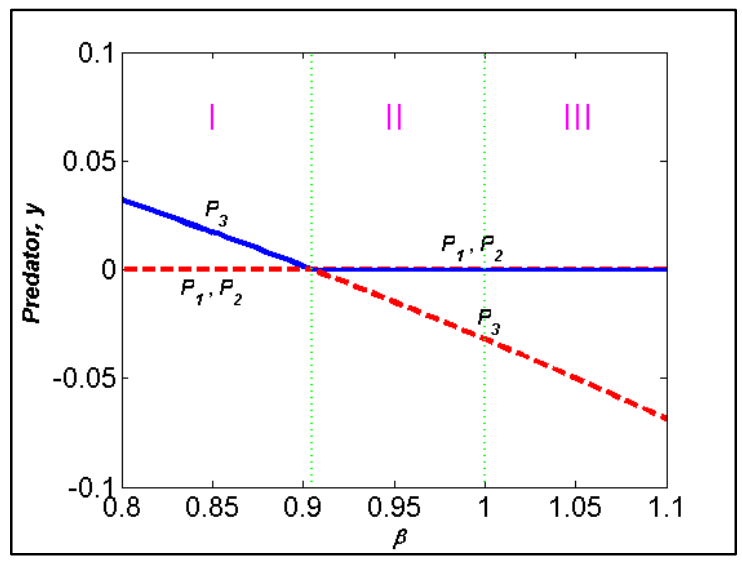

(b)

Fig. 1 Steady-state diagrams of model (3) with respect to the harvesting parameter, $\beta$ with $\alpha=0.1, \sigma=2, \delta=0.1, \varepsilon=0.85$ and $\rho=2$ for (a) prey, $x$, and (b) predator, $y$, respectively.

From Fig. 1(a) and 1(b), there are two transcritical bifurcation points $(\beta=0.905$ and $\beta=1$ ) where the steady-state branches interchange with each other. This is due to the existence of parameter $\beta$ in both characteristic equations (4), (5) and the conditions [i]-[iv]. An intermediate change in parameter $\beta$ will affect the stability and equilibrium of system (3).

From the figures, the population densities of both the prey and predator species decrease as the harvesting activities on prey increase. This situation happens because harvesting activities decrease the number of prey in the system and consequently deplete the food supplies of predator on prey, causing both species to deplete in number. This scenario is described by the solid lines in region (I). In this case, both the species coexist as the steady state of $P_{3}$ is stable. However, when the harvesting activities on prey keep increasing until it exceeds a certain threshold $(\beta=$ 0.905), the steady-state branches of $P_{3}$ and $P_{2}$ interchange with each other where the extinction of the predator species occurs as illustrated in region (II). When the harvesting parameter exceeds the threshold of $\beta=1.0$ as in region (III), the prey species dies out due to the excessive harvesting activities and the extinction of both species occurs in system (3), which is cleary shown by $P_{1}$ in region (III) of Fig. 1(a).

From the bio-ecological perspectives, region (I) in Fig. 1 is encouraging and desirable because the harvesting activities are under control where the fisheries coexist with its environment to sustain a balanced ecosystem. Region (II) is a sign of destructive aquatic ecosystem because the intermediate level of harvesting leads to the extinction of predator species. Prey species continues to grow without the predation pressure and they might produce more toxins and hence deteriorate the environment. The worst real life's scenario is described in region (III) where the over fish farming will lead to the extinction of both fish species. The consequent effects might bring disruption to the entire aquatic ecosystem.

Time series plots are plotted by using the MATLAB software in order to investigate the dynamical behaviors of the system with respect to time as shown in Fig. 2.

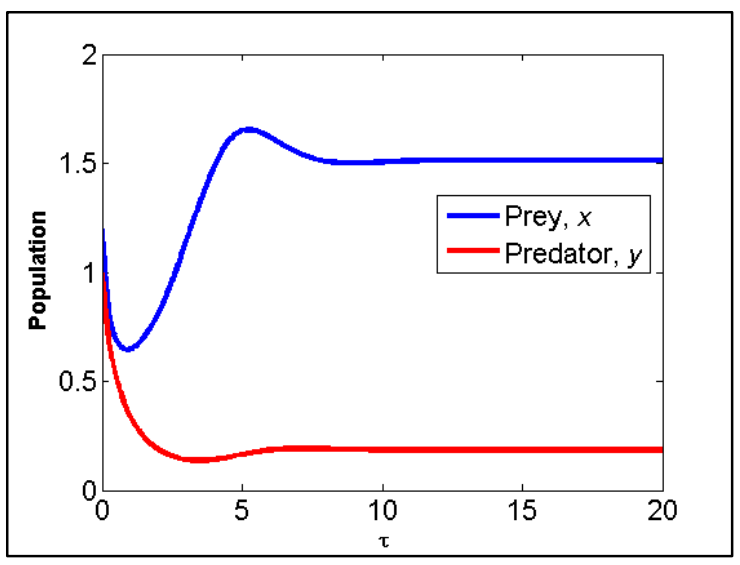

(a) 


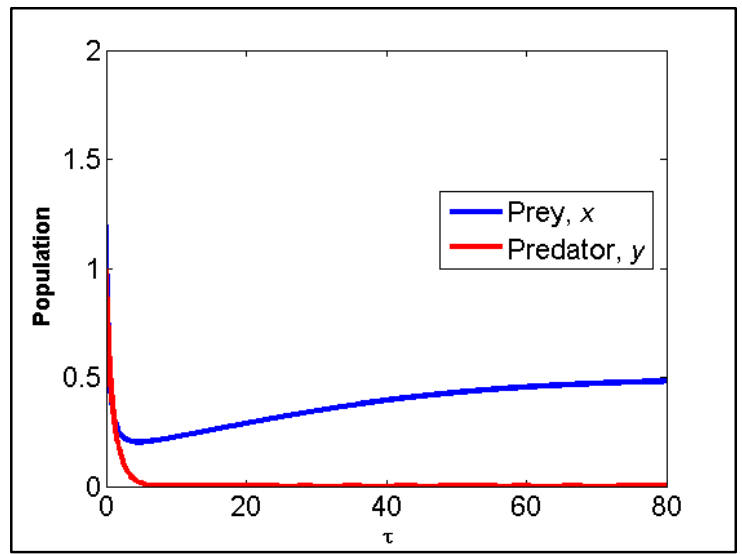

(b)

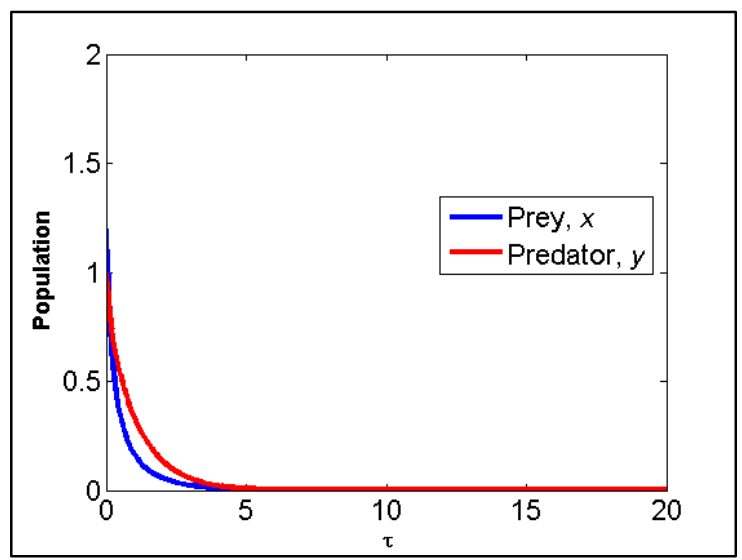

(c)

Fig. 2 Time plots of model (3) with the same parameter values as Fig. 1 and initial conditions of $\left(x_{0}, y_{0}\right)=(1.2,1.0)$ with (a) $\beta=0.1$ (region I), (b) $\beta=0.95$ (region II) and (c) $\beta=1.8$ (region III) respectively.

Referring to Fig. 2(a), the harvesting activities are under control where the harvesting parameter $\beta<0.905$, both prey and predator species coexist. The prey species tends to decrease drastically at the beginning because the prey species are suffering from both harvesting activities and prey-predation. However, after some period, the number of predator reduces causing the prey-predation activities to decrease and therefore, the number of prey species increases again until it is stable at a certain number.

On the other hand, Fig. 2(b) describes the phenomenon and the behaviors of the species at intermediate level of harvesting activities on prey species, $0.905<\beta<1$. The predator population is eradicated from the system after a short period of time. This is because the intermediate level of harvesting activities reduce the number of prey drastically, causing the predator species to lost their food.

From Fig. 2(c), it is observed that both species encounter extinction due to the high level of harvesting activities on prey species. The density of prey species decrease more severely than predator species showing that the harvesting activities have a greater impact on the population if compared to the toxicant parameter in model (3).

Besides that, we are interested to study the influence of the harvesting activities on predator (parameter $\varepsilon$ ) in model (3).

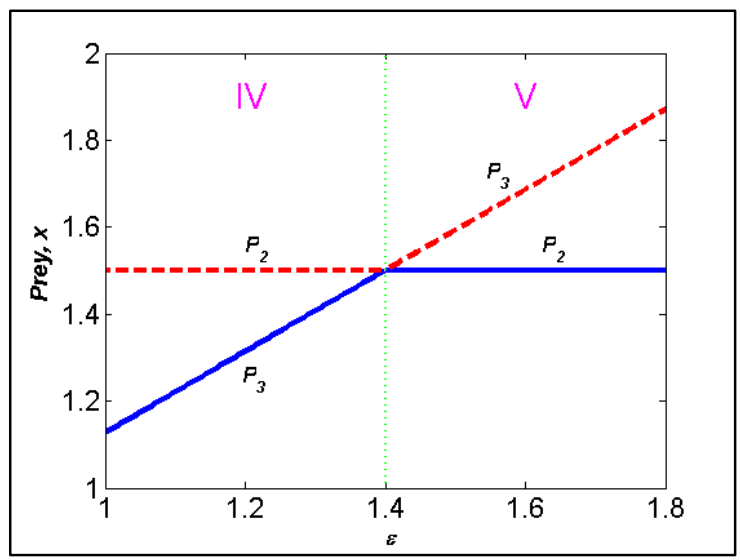

(a)

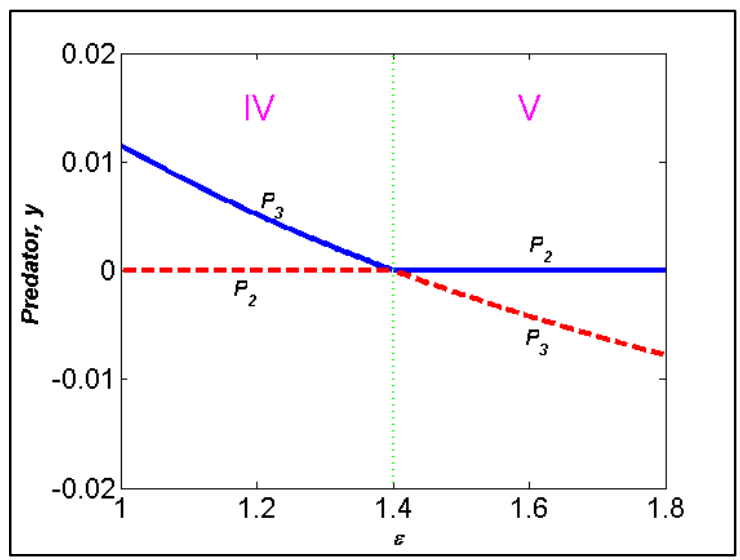

(b)

Fig. 3 Steady-state diagrams of model (3) with respect to the harvesting parameter, $\varepsilon$ with $\alpha=0.1, \beta=0.85, \sigma=2, \delta=0.1$ and $\rho=2$ for (a) prey, $x$ and (b) predator, $y$, respectively.

Fig. 3(a) and 3(b) show the prey population density increases linearly but the predator population decreases gradually as the harvesting parameter $\varepsilon$ increases. This scenario explains the number of predator decreases in result of being harvested. 
Consequently, less prey is being eaten by predator, causing more prey species to release toxin and therefore reduce the number of predator gradually. The predator population decreases due to two rational reasons: the harvesting activities on itself and the toxin released by the prey population that affects the mortality rate of predator species. This scenario is illustrated by the solid lines in both figures where the steady state of $P_{3}$ is stable for $\varepsilon<1.4$ which implies the coexistence state of both species.

In spite of that, when the level of harvesting parameter keeps increasing until it passes through a transcritical bifurcation point of $\varepsilon=1.4$, the steady state branch $P_{3}$ interchanges its stability with $P_{2}$. This simply means that extinction of predator population will occur and only the prey species survives.

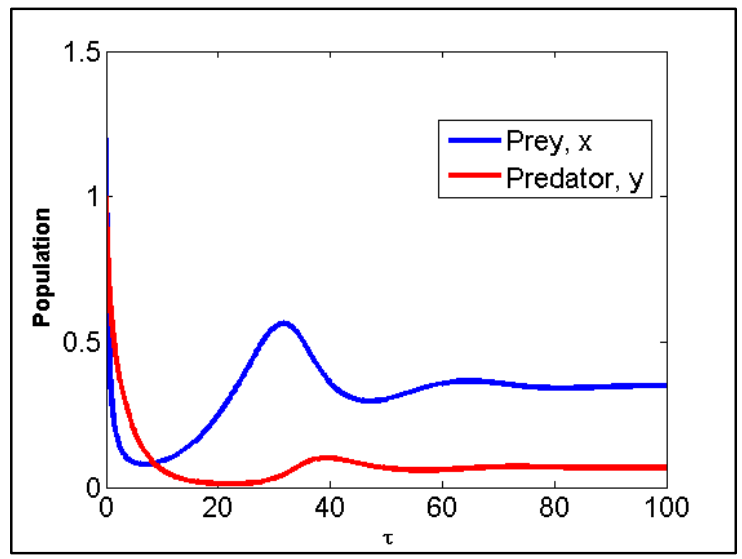

(a)

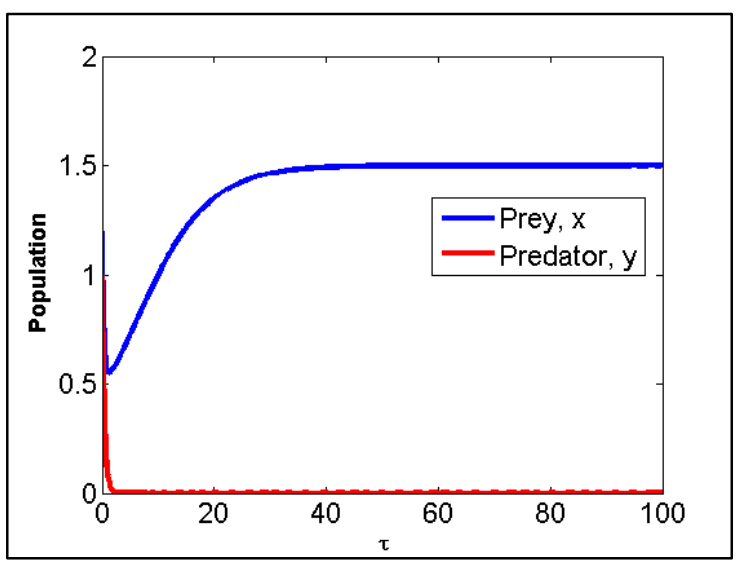

(b)

Fig. 4 Time plots of model (3) with the same parameter values as Fig. 3 and initial conditions of $\left(x_{0}, y_{0}\right)=(1.2,1.0)$ with (a) $\varepsilon=0.2($ region IV) and (b) $\varepsilon=2.6($ region V) respectively.
Fig. 4(a) and 4(b) show time series plots corresponding to bifurcation diagrams in Fig. 3(a) and 3(b). At a low harvesting level $(\varepsilon=0.2)$, both species persist where $P_{3}$ is stable as shown in region (IV) in Fig. 4(a). Both prey-predation activities and the toxin produced by predator may pose the population density of prey to decrease. Excessive level of harvesting activities $(\varepsilon=2.6)$ will lead to the extinction of predator population $\left(P_{2}\right.$ is stable), making the prey population continues to grow until the logistic limiting factor which is illustrated in region (V) in Fig. 4(b).

\section{Conclusion}

In the previous section, a prey-predator system of a fishery model influenced by the harvesting efforts and the toxin released by both species has been studied. Both species are subjected to different harvesting rates. Each species produces toxin to the other species.

The steady states of the model are examined and the stability is discussed. Nonexistence of limit cycle is proved by the Bendixson-Dulac criterion. From the numerical simulations, it may be concluded that at low level of harvesting activities and toxin will guarantee the coexistence of the system but conversely, high level of harvesting activities and toxin will lead to the extinction of the system. In our investigation, we may deduce that the impacts of harvesting activities to the dynamical behaviors of the system are more obvious and crucial if compared to the toxin released. Harvesting parameters seemed to be more critical and influential than the toxin parameters. This is because, by inspection, the toxicant parameters $\sigma$ and $\rho$ are not directly involved in the characteristic equations (4) and (5), causing it to be less influential to the stability of the equilibrium or the system if compared to the harvesting parameters of $\beta$ and $\varepsilon$.

In a nutshell, rational approaches on the sustainable use of fishery resources and ecosystem heath such as the sustainable fishing, restoring collapsed fisheries and setting up marine protected areas should be inculcated. Illegal fishing and over harvesting on fisheries should be avoided to allow future generations to benefit from marine resources. 


\section{Acknowledgements}

The present research is supported by the Fundamental Research Grant Scheme, Ministry of Higher Education Malaysia Vot number 1563 and Universiti Tun Hussein Onn Malaysia.

\section{References}

[1] Hallam, T.G., Clark, C.E. and Lassiter, R.R. (1983). "Effects of Toxicants on Populations: A Qualitative Approach I. Equilibrium Environmental Exposure" in Ecological Modelling, Vol. 18. pp. 291304.

[2] Hallam, T.G., Clark, C.E. and Jordan, G.S. (1983). "Effects of Toxicants on Populations: A Qualitative Approach II. First Order Kinetics" in J. Math. Biology, Vol. 18. pp. 25-37.

[3] Pal, A.K. and Samanta, G.P. (2010). "A Single Species Population in a Polluted Environment" in International Journal of Biomathematics, Vol. 3. No. 2. pp. 187204.

[4] Hallam, T.G. and De Luna, J.T. (1984). "Effects of Toxicants on Populations: A Qualitative Approach II. Environmental and Food Chain Pathways" in J. Math. Biology, Vol. 109. pp. 411-429.

[5] Freedman, H.I. and Shukla, J.B. (1991). "Models for the Effect of Toxicant in Single Species and Predator Prey Systems" in J. Math. Biology, Vol. 30. pp. 15-30.

[6] Chattopadhyay, J. (1996). "Effect of Toxic Substances on A Two Species Competitive System" in Ecological Modelling, Vol. 84. pp. 287-289.

[7] Samanta, G.P. (2010). "A Two Species Competitive System under the Influence of Toxic Substances" in Applied Mathematics and Computation, Vol. 216. pp. 291-299.

[8] Huang, Q., Parshotam L., Wang H., Bampfylde, C. and Lewis, Mark.A. (2013). "A Model for the Impact of Contaminants on Fish Population Dynamics" in Journal of Theoretical Biology, Vol. 334. pp. 71-79.

[9] Scott, G.R. and Sloman, K.A. (2004). "The Effects of Environmental Pollutants on Complex Fish Behaviour: Integradting Behavioural and Physiollogical Indicator of Toxicity" in Aquatic Toxicology, Vol. 68. pp. 369-392.

[10] Huang, Q., Wang H., and Lewis, Mark.A. (2015). "The Impact of Environmental Toxins on Predator Prey Dynamics" in Journal of Theoretical Biology, Vol. 378. pp. 12-30.

[11] Hoekstra, J. and Bergh, J.C.J.M. (2005). "Harvesting and Conservation in A Predator Prey System" in Journal of Economic \& Control, Vol. 29. pp. 10971120.

[12] Kar, T.K. (2003). "Selective harvesting in A Prey Predator Fishery with Time Delay" in Mathematical and Computer Modelling, Vol. 38. pp. 449-458.

[13] Mukhopadhyay, B. and Bhattacharyya, R. (2016). "Effect of Harvesting and Predator Interference in A Model of Two Predators Competing for A Single Prey" in Applied Mathematical Modelling, Vol. 40. pp. 3264-3274.

[14] Ghosh, M., Chandra, P. and Sinha, P. (2002). "A Mathematical Model To Study the Effect of Toxic Chemicals on A Prey Predator Type Fishery" in Journal of Biological System, Vol. 10. No. 2 pp. $97-$ 105.

[15] Das, T., Mukherjee, R.N. and Chaudhuri, K.S. (2009). "Harvesting of A Prey Predator Fishery in the Presence of Toxicity" in Applied Mathematical Modelling, Vol. 33. pp. 2282-2292.

[16] Haque, M. and Sarwardi, S. (2016). "Effect of Toxicity on a Harvested Fishery Model" in Modelling Earth Systems Environment, Vol. 2. pp. 122131.

[17] Kar, T.K. and Chaudhuri, K.S. (2003). "On Non-selective Harvesting of Two Competing Fish Species in the Presence of Toxicity" in Ecological Modelling, Vol. 161. pp. 125-137.

[18] Berryman, A.A. (1992). "The Origins and Evolution of Predator Prey Theory" in Ecology, Vol. 73. No. 5 pp. 1530-1535. 